\title{
BMJ Open Pain education for patients with non- specific low back pain in Nepal: protocol of a feasibility randomised clinical trial (PEN-LBP Trial)
}

Saurab Sharma, ${ }^{1,2}$ Mark P Jensen, ${ }^{3}$ G Lorimer Moseley, ${ }^{4} \mathrm{~J}$ Haxby Abbott ${ }^{2}$

To cite: Sharma S, Jensen MP, Moseley GL, et al. Pain education for patients with non-specific low back pain in Nepal: protocol of a feasibility randomised clinical trial (PEN-LBP Trial). BMJ Open 2018;8:e022423. doi:10.1136/ bmjopen-2018-022423

- Prepublication history and additional material for this paper are available online. To view these files, please visit the journal online (http://dx.doi org/10.1136/bmjopen-2018022423).

Received 16 February 2018 Revised 23 May 2018 Accepted 6 July 2018

Check for updates

(C) Author(s) (or their employer(s)) 2018. Re-use permitted under CC BY. Published by BMJ.

${ }^{1}$ Department of Physiotherapy, Kathmandu University School of Medical Sciences, Dhulikhel Bagmati, Nepal

${ }^{2}$ Centre for Musculoskeletal Outcomes Research, Dunedin School of Medicine, University of Otago, Dunedin, Otago, New Zealand

${ }^{3}$ Department of Rehabilitation Medicine, University of Washington, Seattle,

Washington, USA

${ }^{4}$ School of Health Sciences, University of South Australia Adelaide, South Australia, Australia

Correspondence to

Saurab Sharma

saurabsharma1@gmail.com

\section{ABSTRACT}

Introduction Low back pain (LBP) is the leading cause of years lived with disability in Nepal and elsewhere. Management of LBP that is evidence-based, easily accessible, cost-effective and culturally appropriate is desirable. The primary aim of this feasibility study is to determine if it is feasible to conduct a full randomised clinical trial evaluating the effectiveness of pain education as an intervention for individuals with LBP in Nepal, relative to guideline-based physiotherapy treatment. The findings of the study will inform the planning of a full clinical trial and if any modifications are required to the protocol before undertaking a full trial.

Methods/analysis This protocol describes an assessorblinded feasibility clinical trial investigating feasibility of the pain education intervention in patients with nonspecific LBP in a physiotherapy hospital in Kathmandu, Nepal. Forty patients with LBP will be randomly allocated to either pain education or guideline-based physiotherapy treatment (control). Outcomes will be assessed at baseline and at a 1 week post-treatment. The primary outcomes are related to feasibility, including: (1) participant willingness to participate in a randomised clinical trial, (2) feasibility of assessor blinding, (3) eligibility and recruitment rates, (4) acceptability of screening procedures and random allocation, (5) possible contamination between the groups, (6) intervention credibility, (7) intervention adherence, (8) treatment satisfaction and (9) difficulty in understanding the interventions being provided.

Ethics/dissemination The protocol was approved by Nepal Health Research Council (NHRC; registration number: 422/2017) and University of Otago Human Ethics Committee for Health (registration number: H17/157). The results of the study will be presented at national and international conferences and published in a peerreviewed journal.

Trial registration number NCT03387228; Pre-results.

\section{INTRODUCTION}

Low back pain (LBP) is a highly prevalent health condition worldwide. ${ }^{12}$ It is the leading cause of disability ${ }^{2}$ and imposes huge economic burden to the society in both developed and developing countries. ${ }^{3-6}$ LBP is among the most common health conditions
Strengths and limitations of this study

- Assessor and statistician will be blinded to group allocation of the participants.

- Therapist and patient blinding is not possible in this study because of the nature of the treatments provided.

- Conclusions regarding the effectiveness of the intervention cannot be made because of the feasibility design of the current study.

contributing to years lived with disability in Nepal. ${ }^{2}$ Although the prevalence of LBP is high in Nepal, ethnographic research has noted that LBP-related disability may be low in rural areas, ${ }^{7}$ perhaps due to the very low socioeconomic status of individuals living in rural Nepal, which forces them to keep working despite the presence of pain. Consistent with this idea, another study highlighted that $80 \%$ of people with chronic pain in Nepal continue to work. ${ }^{6}$ However, it is alarming that number of spine surgeries for spinal pain has been increasing in Nepal over the years, ${ }^{8}$ despite lack of evidence supporting efficacy for this treatment. ${ }^{19}$

\section{Interventions for management of LBP}

Many interventions have been investigated for the management of LBP. These include surgery, ${ }^{10}{ }^{11}$ pharmacotherapy, ${ }^{12-17}$ exercises, ${ }^{18-20}$ advice for self-management including advice to remain physically active ${ }^{11}$ and psychological therapies. ${ }^{21}{ }^{22}$ As alluded to earlier, biomedically focused interventions such as surgery and pharmacotherapy are not recommended for a non-specific LBP as the evidence does not support their effectiveness. ${ }^{14}$ Moreover, they are associated with significant risks for adverse events and are costly. ${ }^{5}$

Clinical practice guidelines for LBP recommend self-management including 
reassurance, education and advice to remain active as the first line of care that should be provided to all the patients with LBP. Superficial heat and manual therapy (massage/ manipulative therapy) are recommended for acute LBP, whereas exercise and psychological therapies are recommended for chronic LBP. ${ }^{23-25}$

\section{Pain education for LBP}

Patient education for LBP that has been investigated in randomised controlled trials is basically of two types: biomedical education and pain biology education. ${ }^{26}$ The first refers to educating patients about vertebral anatomy and pathoanatomy of the spine, which has been shown to be ineffective and may even have negative effects on LBP outcomes. ${ }^{26}$ However, the second type of education-pain biology education (hereafter called as 'pain education')- - has been shown to have positive effects on both pain and disability. ${ }^{27}{ }^{28}$ Pain education is structured education programme with specific aims and objectives. ${ }^{29}$ This intervention has a list of target key concepts to be delivered and includes the curriculum contents to deliver the key concepts using up-to-date pain science knowledge, stories and metaphors.

It has been previously hypothesised that this type of education programme using metaphors and stories may be an effective intervention in Nepalese with chronic pain. ${ }^{30}$ However, the pain education materials that have been developed in western cultures are not necessarily valid and equally effective in reducing pain and disability in non-western cultures. Therefore, when developing pain education materials in a newer language or culture, (significant) cultural adaptations of the education materials may be required to make it suitable for the target population, as culturally inappropriate education may not produce desirable results.

Therefore, in order to evaluate the effectiveness of pain education in individuals with non-specific LBP from Nepal, culturally appropriate pain education materials should first be developed for Nepal, specifically. However, it is possible that the adaptations made could potentially reduce its effectiveness. Thus, before testing the adapted pain education in a full clinical trial, a feasibility study is needed in order to determine if a full clinical trial based on the adapted intervention is warranted, or if additional modifications may be needed prior to performing the full trial.

\section{Why the feasibility trial?}

We propose a feasibility trial because: (1) the intervention (ie, pain education) will need significant cultural adaptation, although it has been evaluated for efficacy previously in other languages and western cultures; (2) the adapted intervention has never been investigated for its efficacy or effectiveness before; (3) the population in question (individuals with extremely low socioeconomic status and educational attainment in Nepal) is unique; and (4) a high-quality clinical trial in individuals with LBP has not been conducted in Nepal to our knowledge, and we therefore do not know if a full trial is feasible. The findings from the proposed feasibility study will inform the planning and design of a full trial, if the results indicate that a full trial is warranted.

The results of the full trial will have significant clinical implications for the management of LBP in Nepal and similar cultures, providing empirical evidence if pain education is a viable treatment for the management of LBP, and if it is effective in reducing pain, disability and emotional distress.

\section{Aims and objectives}

The primary aim of the study is to evaluate the feasibility of a full randomised clinical trial (RCT) for assessing the effects of pain education as an intervention for patients with LBP of any duration in a physiotherapy facility in Nepal after developing culturally appropriate, evidencebased pain education materials. The primary objectives of the study are related to feasibility of an RCT, specifically: (1) willingness to participate in an RCT, (2) feasibility of assessor blinding, (3) eligibility and recruitment rates, (4) acceptability of screening procedures and random allocation, (5) possible contamination between the groups, (6) intervention credibility for patients with LBP, (7) intervention adherence, (8) treatment satisfaction and (9) difficulty in understanding the intervention being provided.

\section{METHODS AND ANALYSIS}

\section{Study design and setting}

This is a feasibility study that is being performed to determine if a full RCT can be successfully conducted using the procedures and protocol of the feasibility study, or if modifications of the protocol are needed prior to conducting the full trial. The study findings will inform the design of the full trial, if the trial is found to be feasible. ${ }^{31}$

The definition of a feasibility study highlights the question, 'Can this study be done?'. The Standard Protocol Items: Recommendations for Interventional Trials (SPIRIT) statement, ${ }^{32}$ the Consolidated Standards of Reporting Trials statement extension to pilot and feasibility randomised trial ${ }^{31}$ were followed in the planning of the study and reporting of the protocol.

The study will be an assessor-blinded, two-arm, feasibility RCT. The study is registered in Clinicaltrials.gov (trial registration number: NCT03387228). The study will be conducted in the Sahara Physiotherapy Hospital, Kathmandu, Nepal.

\section{Overview of the study}

Advertisement of the trial will be made in social media, and all the patients with LBP presenting at the study site will be invited to participate. Interested candidates will be screened for eligibility. Eligible patients with non-specific LBP will then be enrolled in the trial and be randomly assigned to one of the two study groups. All the participants in the experimental group will receive pain 
education and those in the control group (CG) will receive guideline-based physiotherapy treatment. All the participants will be assessed at baseline and 1 week following treatment. Details describing the schedule of enrolment, interventions and assessment are presented in table 1, in the manner recommended by SPIRIT checklist. ${ }^{32}$

Table 1 Schedule of enrolment, assessment and interventions

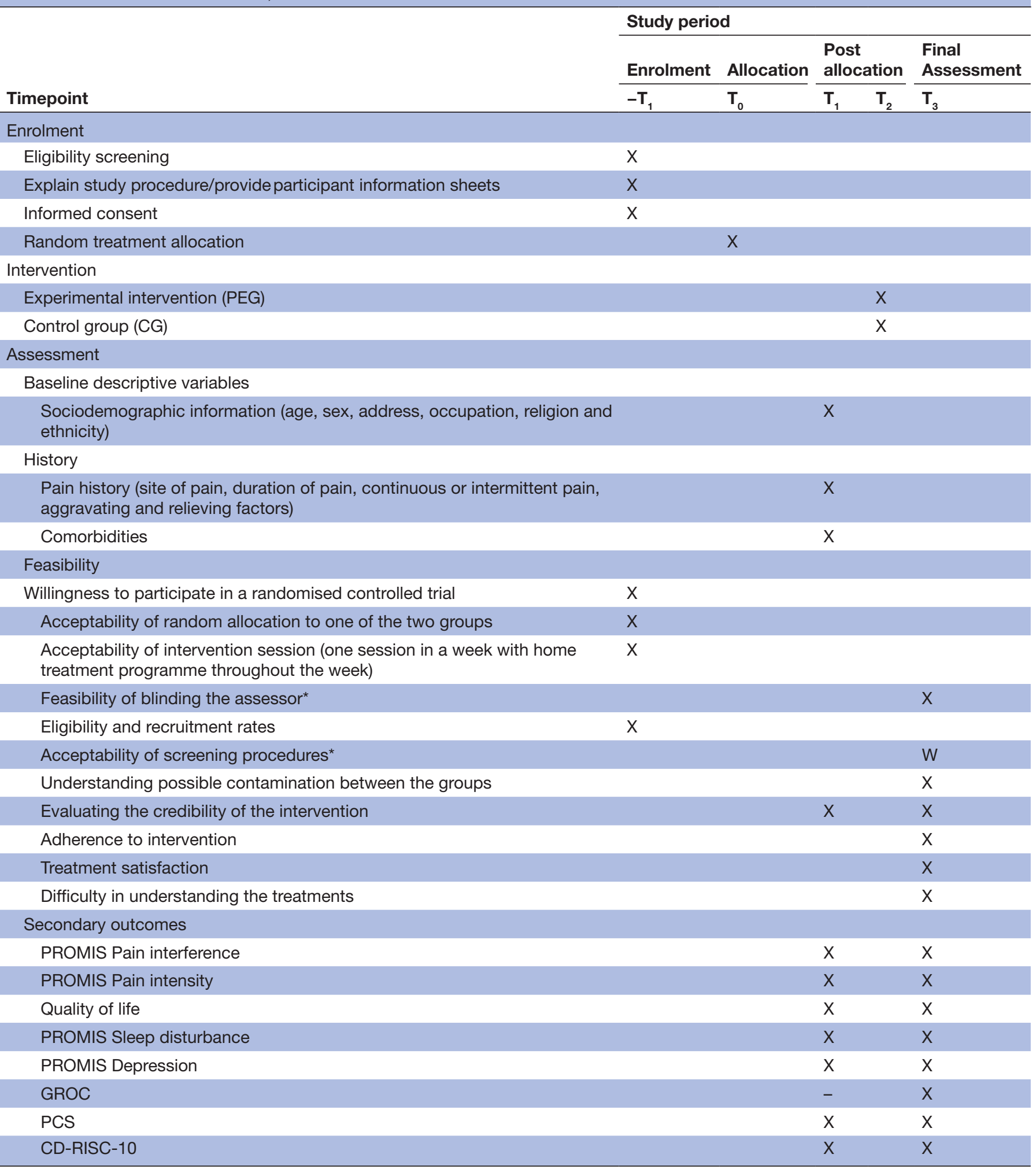

*Assessed by the therapist providing intervention; all other outcomes are assessed by the blinded outcome assessor. CD-RISC-10, 10-item Connor-Davidson Resilience Scale; GROC, Global Rating of Change; PCS, Pain Catastrophizing Scale; PROMIS , Patient-Reported Outcome Measurement Information System; $T_{1}$, enrolment time; $T_{0}$, allocation time; $T_{1}$, baseline assessment (before treatment); $T_{2}$, during treatment; $T_{3}, 1$ week post-treatment; $W$, assessment at the end of every week on Fridays. 


\section{Participants}

Patients with non-specific LBP seeking rehabilitative services at Sahara Physiotherapy Hospital will be invited to participate. Interested patients will be screened by a research assistant (physiotherapist by training) involved in the research.

\section{Inclusion criteria}

Non-specific LBP (LBP other than those excluded, see exclusion criteria below) of any duration with pain primarily localised between T12 and gluteal folds, in patients aged 18 years or older, with average pain intensity reported as moderate, severe, or very severe on a Patient-Reported Outcome Measurement Information System (PROMIS) five-point PROMIS Pain Intensity Short-form Scale $^{33}$ over the past week, and who is a Nepalese and is able to understand and speak Nepali fluently will be included in the study.

\section{Exclusion criteria}

Participants with likely specific causes of LBP will be excluded using a triage procedure as suggested by Bardin and colleagues. ${ }^{34}$ This includes exclusion of participants having history of prolonged use of corticosteroid, history of malignancy, recent history of fever or chills, history of other diseases associated with compromise in immune system, history of recent spinal surgery or dental procedures, recent history of trauma to spine or a fracture of a spine, history of bladder and bowel dysfunction, history of perineal or saddle anaesthesia and history of weakness of lower extremity or loss of sensation in lower extremity. Additionally, current pregnancy and history of diagnosed mental health conditions that would limit adherence to the trial procedures will be excluded.

\section{Sample size}

For a feasibility study, it is inappropriate to calculate sample size based on desired statistical power to detect a treatment effect, ${ }^{35}$ because the primary aim of the study is to assess if a full trial can or should be conducted. Feasibility outcomes are descriptive in nature; therefore, inferential statistics regarding treatment effects will not be computed. To achieve the primary objectives related to feasibility outcomes, the research team estimated that 40 participants would be adequate. ${ }^{36}$ Twenty patients will be randomly allocated to each treatment condition.

\section{Participant screening and recruitment}

Consecutive participants with non-specific LBP will be invited to participate in this study. The study purpose and procedures will be described to potential participants. This will include information about the benefits and potential harms of the intervention, the time required for the completion of the study, follow-up duration, voluntariness of participation, cost of participation and the rights to withdraw from the study at any point. A study information sheet will be provided to all potential participants.

If the potential participants are interested in participating, they will be screened for eligibility by a research assistant who is a physiotherapist. If the participants are found eligible, informed consent will be obtained. For those who cannot sign the consent, a witness will sign on their behalf, or the study participant will provide a thumb print on the form for those who cannot write or sign the form as per the ethical guidelines provided by Nepal Health Research Council (NHRC). We will include uneducated patients who cannot sign an informed consent in order to increase the inclusion of uneducated or low education group, given that $31 \%$ people in Nepal who are 5 years old or more cannot read and write. ${ }^{37}$ Additionally, exploration of feasibility of pain education in those with no schooling or low educational attainment is important in order to inform clinical practice.

Participants will be informed that they will receive one of the two treatments randomly. It will be highlighted that both of the treatment options are thought to be effective for LBP and that the goal of the main study is to compare the interventions; however, the current study will more specifically evaluate the feasibility of such a study.

\section{Group allocation, randomisation and blinding}

Random number sequence, in random blocks of 4 and 6 , will be generated using www.randomization.com, by a researcher (JHA) who is not involved in recruitment process. Allocation concealment will be performed using opaque, sealed envelopes. The participants will be allocated to one of the two groups by a hospital staff member who is not the assessor. The two groups will be: pain education group (PEG) and CG.

\section{Intervention}

The Template for Intervention Description and Replication Checklist was followed when planning the study intervention. ${ }^{38} 39$ Manuals of standard operating procedures will be followed during the delivery of the interventions in both the groups. This will ensure treatment uniformity and fidelity. It is not possible to blind the intervention providers based on the design of the study.

Participants in intervention group (PEG) will receive detailed pain education as described in the next paragraph, and those in the CG will receive guideline-based physiotherapy treatment. After the completion of the post-treatment assessment at 1 week, study participants in both the groups will receive the treatment being provided by physiotherapists at Sahara Physiotherapy Hospital. Participants in both the groups will be encouraged not to seek for other medical care for LBP during the 1-week study period, unless they have to. If they do undertake other forms of treatment, they will be requested to report this during the follow-up assessment, and this will be recorded.

\section{Pain education group}

The pain education will be delivered to the PEG only. We will use the pain education handouts in Nepali for Nepalese with LBP based on the resources developed by Moseley and Butler, called Explain Pain. ${ }^{29} 40$ it 
has evolved in over 15 years $^{42}$ and undergone changes and advances. ${ }^{29} 40414344$ Pain education is delivered to provide reassurance, which means removal of fear and concerns about illness. ${ }^{45}$ Reassurance is among the core aspects of management of patients with non-specific problems such as LBP. ${ }^{46}$ Although there are no gold standard ways to provide reassurance and alleviate fear and concerns about a disease or its consequences, empathy and collaboration are thought to play important role. ${ }^{47}$ The Pain education intervention will be provided by the lead investigator (SS) who is trained in the delivery of this treatment and has about 10 years of experience in the management of musculoskeletal disorders, including LBP.

In order to develop the pain education resources in Nepali, the first step is the development of curriculum for pain education for patients with $\mathrm{LBP}^{29}$ in Nepal. The curriculum was first outlined in English (by SS) in the similar manner recommended by the developers ${ }^{29}$ and was reviewed and approved by both the developers (see online supplementary appendix 1). Based on the curriculum, the pain education materials and patient handouts were created (by SS) in Nepali. The clinical cases and pain stories that were compiled are actual stories collected from patients and clinicians in Nepal but will be anonymously shared to provide reasoning based on contemporary pain biology knowledge. ${ }^{29}$ Pictures that are found in Explain Pain resource materials ${ }^{29} 4041$ were adapted in the Nepali version.

The pain education handout and materials that are produced in Nepali is proofread and will first be pretested in 5-10 Nepalese with LBP as needed, and corrected, if necessary, before using in the feasibility study participants. The final handouts will then be printed for the use in the current study. This adapted process will ensure that the Nepali pain education materials produced are valid and culturally appropriate. However, for the purpose of the full trial, the difficulty in understanding the treatment will be assessed in the current feasibility study, and any modifications required will be made.

\section{Dosage of the PEG intervention}

A single approximately 1 hour pain education will be delivered to the PEG, because evidence indicates that: (1) interventions as brief as $5 \mathrm{~min}$ have been shown to have reassuring effects lasting for up to a year, ${ }^{44}$ and a single consultation has been found to be as reassuring as the multiple session interventions. ${ }^{47}$ We prefer a single session treatment over multiple session treatment, because for many patients in rural Nepal, it is difficult to deliver a multiple session treatment in reality. Thus, we plan to provide a single session delivering key concepts of Explain Pain, which will be reinforced by providing patient handouts to look at and read at home. In fact, a 1-hour session should be adequate to cover the key Explain Pain concepts by keeping the content simple and jargon free. Use of a plain language in the health-related education is important to adapt in low health literacy. ${ }^{48}$

\section{Home advice for PEG group}

A printed handout of the pain education material will be provided only to the study participants in the PEG. Participants will be advised to read them, and perform physical activity including walking for approximately $30 \mathrm{~min}$. Education accompanied by written information has been reported to yield the largest effects on fear component of emotional distress. ${ }^{47}$ However, education level of Nepalese is low $(65 \%) ;{ }^{37}$ we will adapt the written materials to incorporate many more images than text.

For those who cannot read, the family member(s) will be encouraged to read out the materials to them. To complement the written materials, we will also provide an audio-recording of the pain education session to the patients as an online URL link to Nepali patient education material stored in the cloud or will be copied to their smart phones or both for those who have the facilities to use them. The pain education advice will be directed towards reducing brain's perception of movement and exercise as a threat encouraging participants to slowly pace the movement, physical activity and exercise. This is thought to desensitise the sensitive nervous system and improve function. A written instruction to perform general exercises and physical activity will be sent to the participants. Participants will be discouraged to use a lumbar corset and rest as coping strategy, whereas physical activity and return to work will be encouraged. A reminder to perform home exercises will be sent to all participants for a total of 5 days in the week.

\section{Control group}

The intervention will be provided by the physiotherapists working at the study site. Treatment integrity in the CG will be improved by providing an interactive seminar to all the physiotherapists delivering CG intervention by the lead researcher. The seminar will incorporate evidence-based information, including assessment and management of LBP based on the current recommendations from clinical practice guidelines. ${ }^{23}{ }^{49-51}$ Research articles and simplified evidence-based summary will be provided to the physiotherapists before the interactive seminar for self-study. At the end of the seminar, a brief multiple-choice quiz will be conducted for the study physiotherapists that will assess evidence-based management of LBP. The therapists will need to score a minimum of $80 \%$ before they deliver treatment to the CG.

\section{Intervention in the CG}

The control participants will receive physiotherapy care based on the recent clinical practice guidelines from three different western countries: (1) American College of Physicians, USA (2017), ${ }^{23}$ (2) National Institute for Health and Care Excellence, UK (2016) ${ }^{49} 51$ and (3) Toward Optimized Practice, Canada (2015) ${ }^{50}$ These guidelines are used because they are recent and highly regarded. We did not find any evidence-based clinical practice guidelines for the management of LBP in Nepal or other developing countries. The CG interventions were derived by 
comparing the recommendations made by each of the three guidelines on specific management strategies. The CG treatment components were selected if: (1) two or more guidelines recommended the treatment component, (2) all the authors of the current study agreed that the component should be included, (3) the component was culturally acceptable and feasible to deliver in Nepal, (4) the component was determined to be appropriate to deliver at first contact and (5) the total duration of all of the components would sum up to 1 hour (approximately) to make the contact hour comparable with the approximately 1 hour of pain education that would be provided to the experimental group. Similarities and differences in the clinical practice guidelines across the countries are common and are influenced highly from the experience of the expert committee responsible for developing a clinical practice guideline and local practice trends. ${ }^{52}$ For this reason, we chose to adapt the recommendations made in the available guidelines to fit with the expertise of the local physiotherapist, practice trends that are widely accepted in Nepal and the feasibility of delivering the treatment within the context of the study.

Thus, the CG intervention will contain of: (1) education (advice to remain active, education about prognosis of LBP for acute LBP and avoid bed rest and braces) ${ }^{235051}$ for $10-15$ min, including time spent to listen to each participant's pain story; (2) back massage ${ }^{2351}$ for about $10 \mathrm{~min}$; (3) superficial heat ${ }^{235}$ for $10-15 \mathrm{~min}$; and (4) static cycling or (treadmill) walking with the aim to promote physical activity ${ }^{230} 51$ including any rest period for a total of approximately $20 \mathrm{~min}$. Although superficial heat is recommended in the acute/subacute LBP, ${ }^{23}{ }^{50}$ we included this as a common treatment for all types of LBP including chronic LBP, which could be a part of self-management. Any forms of electrotherapy and acupuncture will not be offered to the study participants. ${ }^{49}$ The control intervention will strictly exclude the use of pain biology education.

\section{Dosage of intervention for the $\mathrm{CG}$}

The control intervention will last for 1 hour to match the experimental group.

\section{Home advice for the CG}

Participants will be advised to self-manage their back pain based on the information provided. Home exercise leaflet with emphasis on the value of exercise to increase strength and endurance, followed by a 30 min walking. Advice preceding the exercises will state that exercises are needed to keep you strong, healthy and pain free. A written instruction to perform general exercises and physical activity will be sent to the participants. Participants will be discouraged to use a lumbar corset and rest as coping strategy, and return to work and physical activity will be encouraged in the control participants, as they are in the experimental group. A reminder to perform home exercises will be sent to all the participants for 5 days during the week.

\section{Outcome measures}

The details of primary feasibility outcomes are presented in table 2.

All but one secondary outcome measures have been shown to be reliable and valid in Nepali populations. The measure of quality of life $(\mathrm{QOL})$ is not yet validated at the time of writing the protocol; however, we have included it as a secondary outcome measure, because assessment of QOL is a recommended measure by core outcome sets in clinical trials for $\mathrm{LBP}{ }^{53}$ We hypothesise that this measure is comprehensible and will show adequate validity in the Nepalese sample. We will evaluate the validity of the measure before using it in the full clinical trial.

A research assistant will interview all the study participants to make the study procedures consistent and to allow for the inclusion of participants with little or no education. The interviews will be administered by a physiotherapist who will be blind to group assignment. Secondary measures include the four PROMIS shortform measures assessing pain interference ${ }^{54}$ pain intensity ${ }^{54}$ sleep disturbance,${ }^{54}$ and depression, ${ }^{54}$ as well as the 13-item Pain Catastrophizing Scale, ${ }^{55}$ Connor-Davidson Resilience Scale ${ }^{56}$ Global Rating of Change ${ }^{57} 58$ and a QOL scale. All the items in each PROMIS measure will be summed to obtain raw scores for each scale. The raw scores of each measure will then be converted to T-scores, with a mean of 50 and SD of 10 and recorded (www. assessmentcenter.net). Details of the measures with their measurement properties are presented in table 3 .

The risk of adverse events in both the groups are very low. Participants will be asked to choose the amount of home exercises (such as walking) they will perform based on a level that is comfortable. Participants will be asked to change the duration and/or pace of exercises if they feel the initial level is too high. ${ }^{59}$ Participants will be asked to record any adverse events that occur and report these to the researcher. Adverse events in both the groups will be reported and compared between the groups.

\section{Additional measures}

Additional questionnaires will be administered to obtain data related to: (1) sociodemographic information (age, sex, education level, employment status, income, religion and ethnicity); (2) pain history, including duration of pain, aggravating and relieving factors, other associated comorbidities; and (3) pain location using pain drawings. Other information such as resources required to conduct the trial (eg, cost) and time required to complete the recruitment of desired number of participants will also be recorded. ${ }^{60}$ Total duration of home exercises in each group will also be recorded.

\section{Criteria for feasibility}

The results of this feasibility trial will indicate if the study as designed is feasible, which will inform the decision of progressing to a full trial with the recommendations. The decision will be one of the following: (1) do not proceed 


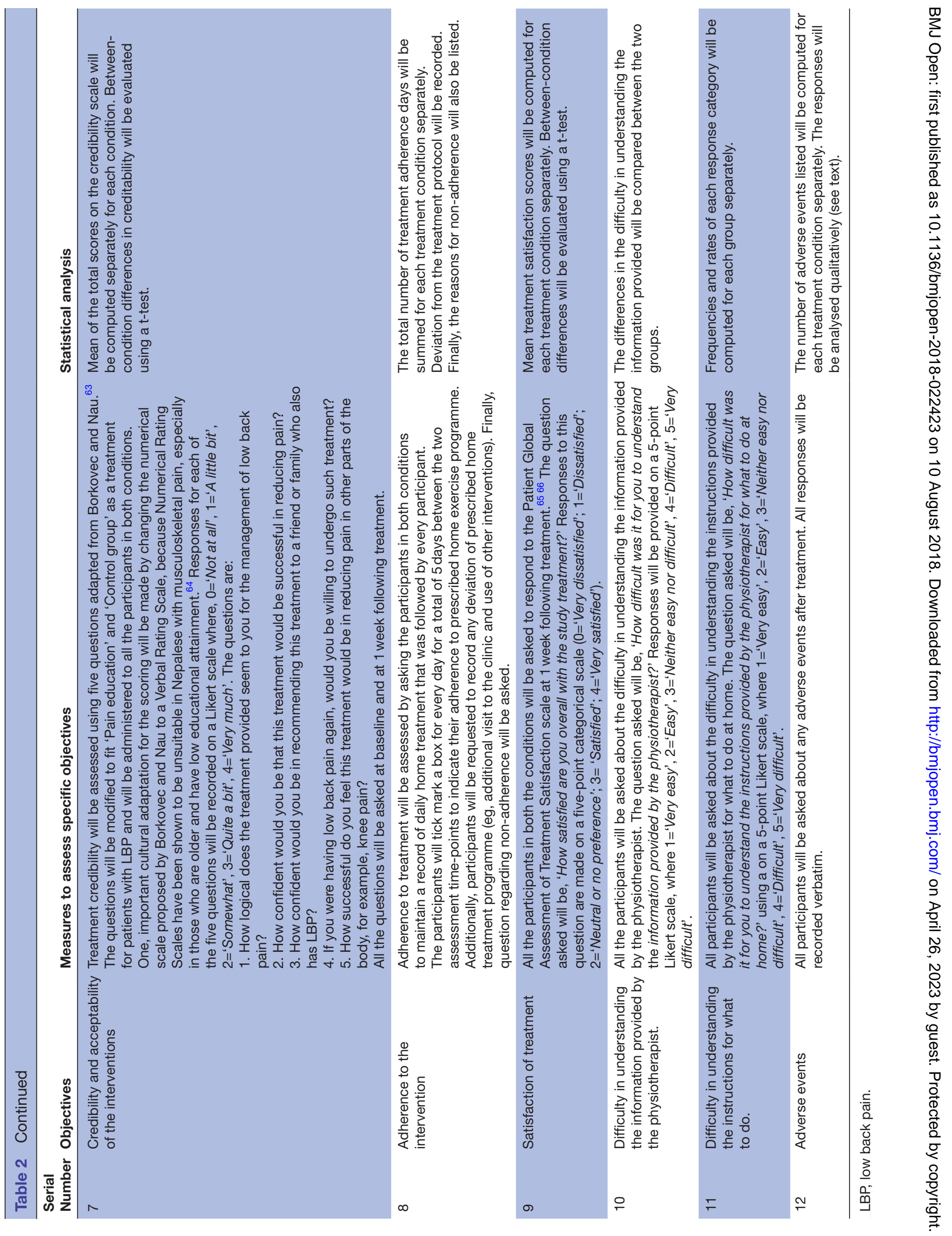




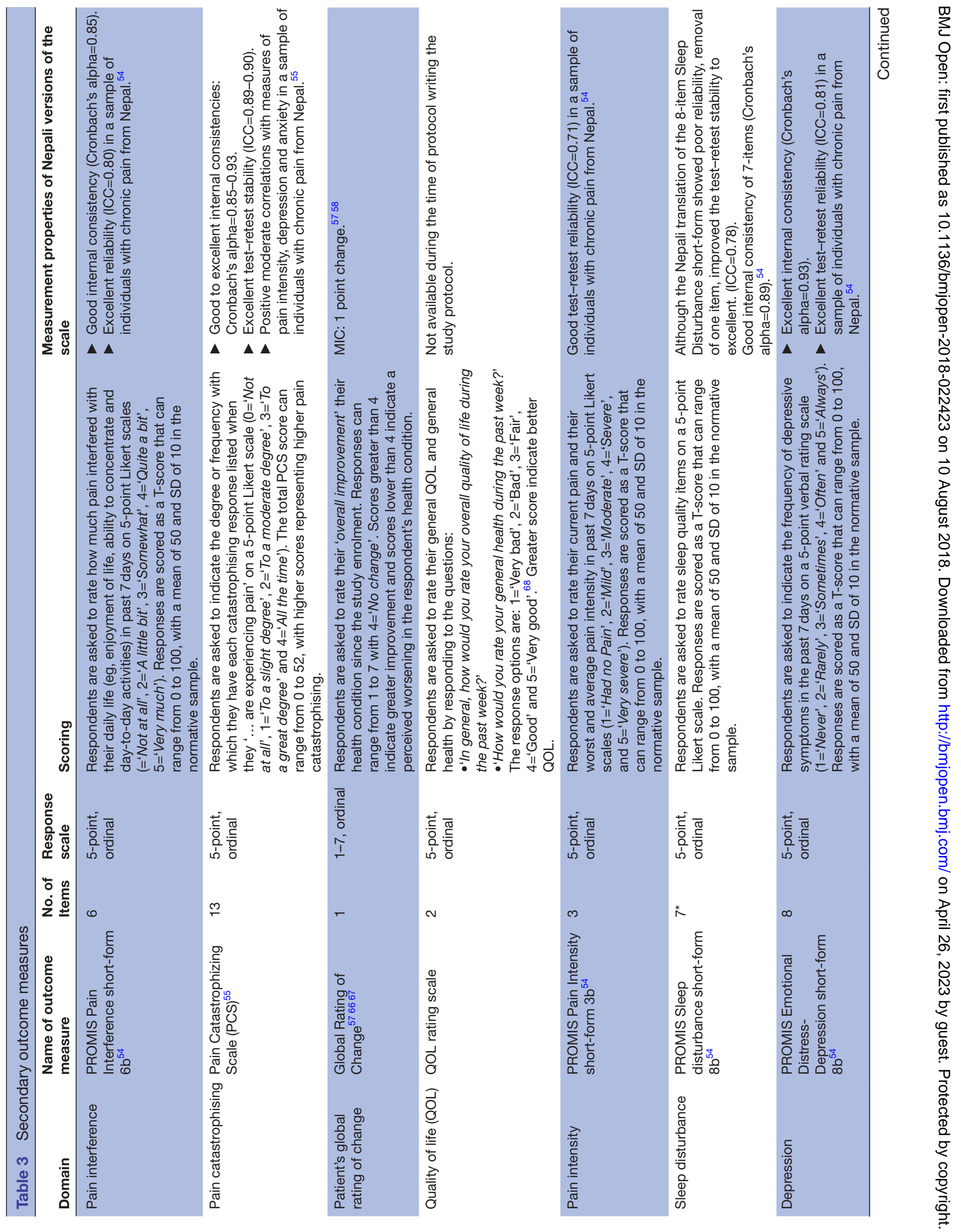




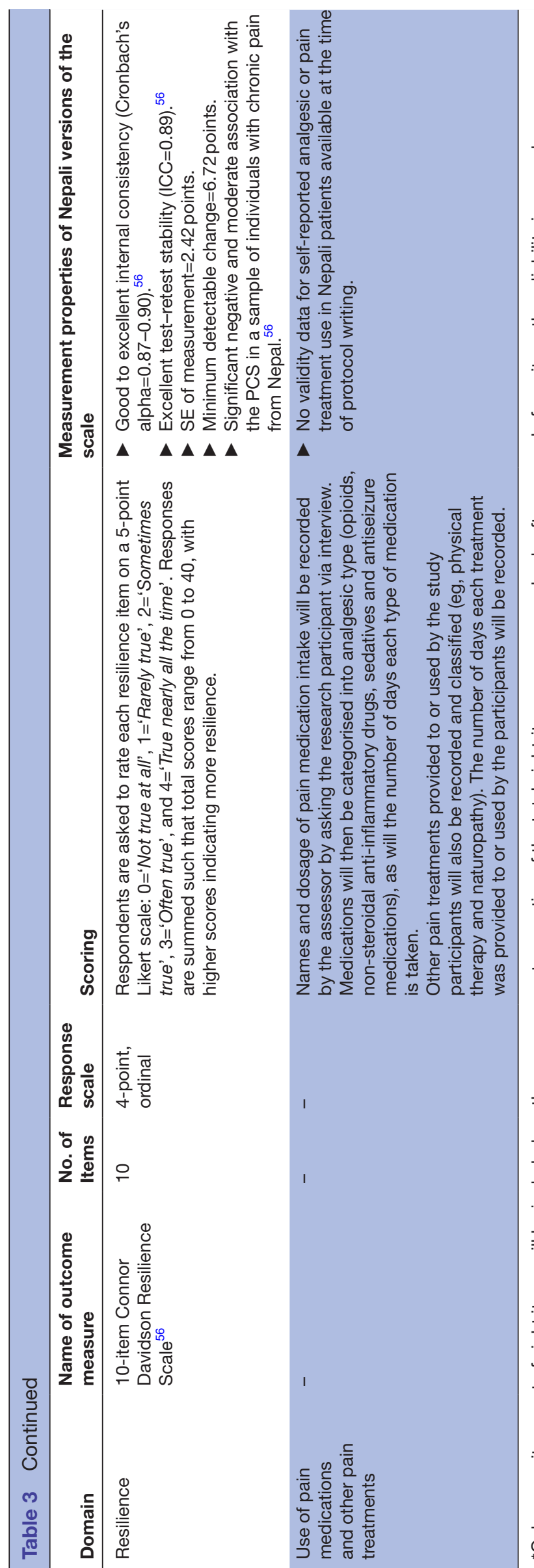

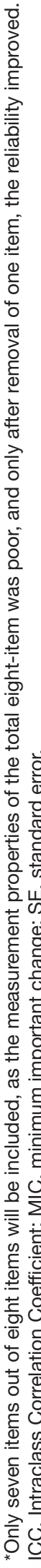

to a full trial if any preplanned changes may not help improve the feasibility; (2) modify the protocol further prior to conducting a full trial; (3) continue with the full trial using the same procedures used in the feasibility trial without modifications; however, monitor the study procedures closely; and (4) continue without modifications, as it is in the feasibility trial, close monitoring is not required. ${ }^{61}$ The criteria for the feasibility are presented in table 4 .

\section{Limitation of the study}

As this is a feasibility study, the results of the current study will not provide findings regarding the efficacy of the interventions being tested. The study will only evaluate if this research study is viable as a full trial and inform any recommendations for modification of the protocols for the full trial resulting from the findings of this feasibility trial.

\section{Plan for supervision and monitoring}

The study will be conducted and monitored by the lead investigator under the supervision of the coauthors (JHA and MPJ), with assistance from research assis$\operatorname{tant}(\mathrm{s})$. All the ethical principles as provided by Declaration of Helsinki will be followed by all the members of this research throughout the study. The investigators will not violate any of the rules and ethical principles of NHRC. Monitoring for the NHRC ethical principles will be regulated by the primary investigator and followed by all researchers and research assistants involved in the study.

\section{Plan for data integrity and management}

The research data will be collected by a research assistant who will be trained to collect the research data and manage the data by compiling in a file for individual patient. Participant identifiers (including name, address and contact information) will be removed from the research data and will be stored separately. Data will be entered in Microsoft Excel. Identification of the groups as intervention and CG will be removed from the excel sheet. Research data will be monitored weekly by scrutinising entered data. Any errors in entry will be identified (if any) and amended. Consent forms will be scanned and stored in password-protected computers of the lead researcher and at the University of Otago along with other research data files.

\section{Data analysis plan}

Descriptive statistics will be computed to describe the baseline and demographic characteristics of the study participants. As it is a feasibility study, level of significance and hypothesis testing regarding treatment efficacy will not be performed. Effect sizes representing betweengroup differences in change in the primary and secondary outcomes will be computed, but these effect sizes will not be considered as a criteria for sample size estimation for the full trial, nor as a criteria to proceed to the full trial, because of the inadequate power of the current feasibility study. Treatment effects for the secondary outcome 


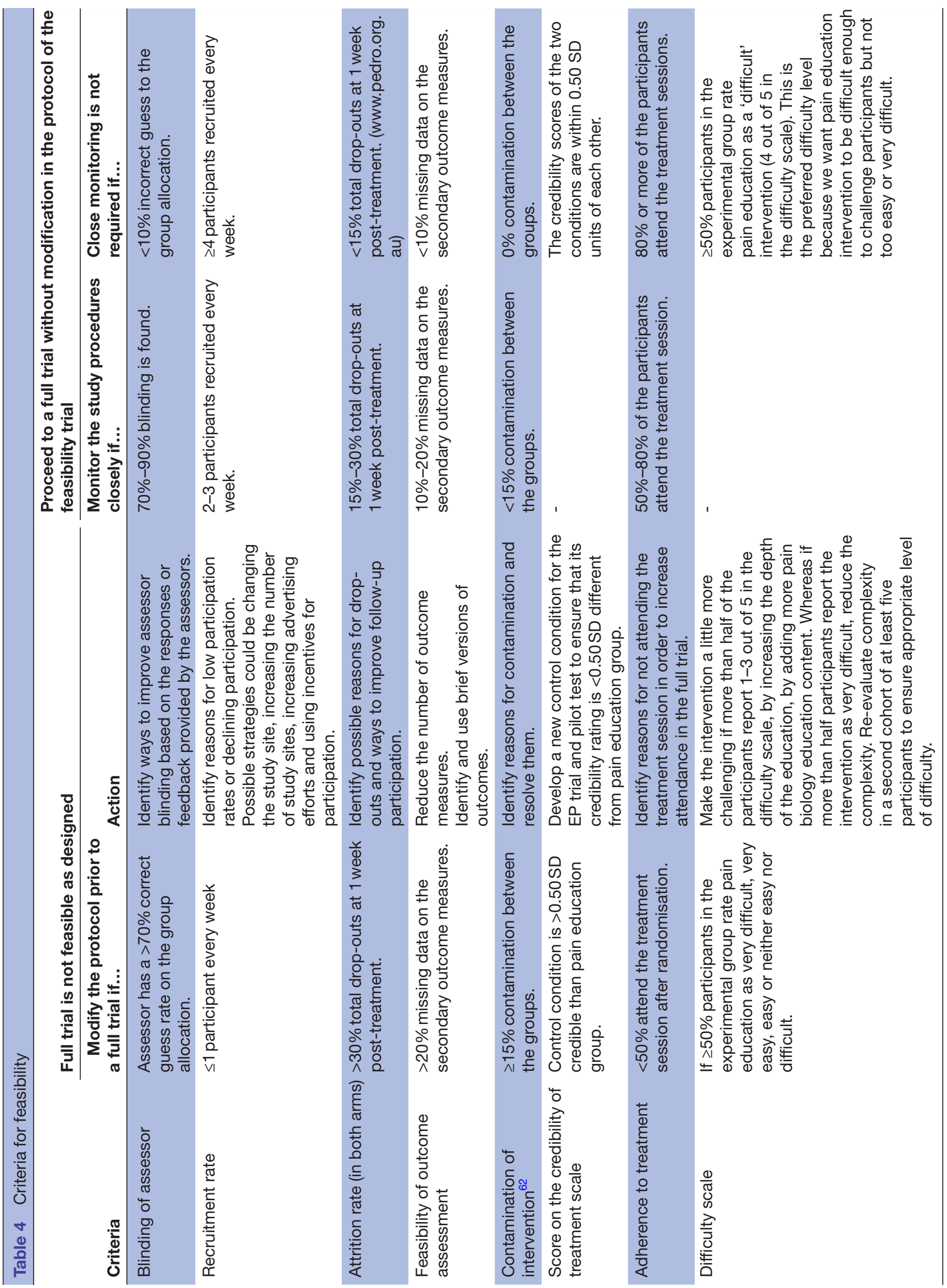


measures will be presented as means, SD and CIs of the means. Difference between the mean scores of each secondary outcome will be compared with the minimum important change (MIC) values of the outcome measures, if the MIC scores are available. The analysis plans of the primary feasibility objectives are described in table 2 .

\section{Patient and public involvement}

The research question was informed by the clinical observation that many patients from rural Nepal showed significant improvement in their LBP outcomes after reassurance and advice to remain physically active. We therefore designed this trial to assess the feasibility of conducting a study to compare the effectiveness of pain education and structured guideline-based physiotherapy treatment in Nepalese people with non-specific LBP.

Patients were not involved in the design of the study protocol but were directly involved in the development of Nepali versions of outcome measures. Patients will also provide feedback and comments in the Nepali pain education materials during pretesting before using it in the feasibility study. Similarly, the development of Nepali Pain Education materials have incorporated real pain-related stories of Nepalese living with pain. The name and identity of all patients were kept confidential. Any information that discloses identity of the patients were excluded in the written pain education booklet.

During the initial assessment, all participants will be asked if they would like to know about the results of the study. A plain language summary of the study results will be written both in English and Nepali, which will be published online. The principal investigator of the study (SS) will also post an audio summary of the research results online for those who cannot read. The link of these will be sent to the participants as text messages.

\section{ETHICS AND DISSEMINATION}

The results of the study will be presented at national and international conferences and published in a peer-reviewed journal.

\section{Twitter link_physio}

Acknowledgements The authors would like to thank Associate Professor David Butler and Tim Cocks from Neuro-Orthopedic Institute (NOI) in Adelaide for their input during the development of curriculum for explaining pain for this study. Dr Adrian Traeger (University of Sydney) provided valuable suggestions in the earlier version of the protocol. Finally, authors would also like to thank all the patients who agreed to use their pain stories in this Pain Education programme.

Contributors SS, JHA and MPJ conceived the idea of the trial. Trial was designed by all authors. SS prepared the first draft of the protocol. Successive drafts were edited by JHA, MPJ and GLM. The final version of the protocol was approved by all the authors.

Funding The authors have not declared a specific grant for this research from any funding agency in the public, commercial or not-for-profit sectors.

Competing interests SS is supported by University of Otago Doctoral Research Grant. GLM has received support from Pfizer, AIA Australia, Gallagher Bassett, Kaiser Permanente USA, Port Adelaide Football Club, Arsenal Football Club and the International Olympic Committee. GLM receives royalties for books on pain and rehabilitation, including the text on which the content for the proposed intervention was based. He also receives speaker fees for lectures on pain and rehabilitation. Other authors report no competing interests.

Patient consent Obtained.

Ethics approval The protocol was approved by Nepal Health Research Council (ref number 422/2017) and University of Otago Human Research Committee (Health) (ref number $\mathrm{H} 17 / 157$ ).

Provenance and peer review Not commissioned; externally peer reviewed.

Open access This is an open access article distributed in accordance with the Creative Commons Attribution 4.0 Unported (CC BY 4.0) license, which permits others to copy, redistribute, remix, transform and build upon this work for any purpose, provided the original work is properly cited, a link to the licence is given, and indication of whether changes were made. See: https://creativecommons.org/ licenses/by/4.0/.

\section{REFERENCES}

1. Balagué $F$, Mannion AF, Pellisé $F$, et al. Non-specific low back pain. Lancet 2012;379:482-91.

2. GBD 2016 Disease and Injury Incidence and Prevalence Collaborators. Global, regional, and national incidence, prevalence, and years lived with disability for 328 diseases and injuries for 195 countries, 1990-2016: a systematic analysis for the Global Burden of Disease Study 2016. Lancet 2017;390:1211-59.

3. Walker BF, Muller R, Grant WD. Low back pain in Australian adults: the economic burden. Asia Pac J Public Health 2003;15:79-87.

4. Hong J, Reed C, Novick D, et al. Costs associated with treatment of chronic low back pain: an analysis of the UK General Practice Research Database. Spine 2013;38:75-82.

5. Machado GC, Maher CG, Ferreira PH, et al. Trends, Complications, and Costs for Hospital Admission and Surgery for Lumbar Spinal Stenosis. Spine 2017;42:1737-43.

6. Bhattarai B, Pokhrel PK, Tripathi M, et al. Chronic pain and cost: an epidemiological study in the communities of Sunsari district of Nepal. Nepal Med Coll J 2007;9:6-11.

7. Anderson RT. An orthopedic ethnography in rural Nepal. Med Anthropol 1984;8:46-59.

8. Bajracharya S, Singh M, Singh GK, et al. Clinico-epidemiological study of spinal injuries in a predominantly rural population of eastern Nepal: A 10 years' analysis. Indian J Orthop 2007;41:286-9.

9. Deyo RA, Nachemson A, Mirza SK. Spinal-fusion surgery - the case for restraint. N Engl J Med 2004;350:722-6.

10. Fritsch CG, Ferreira ML, Maher CG, et al. The clinical course of pain and disability following surgery for spinal stenosis: a systematic review and meta-analysis of cohort studies. Eur Spine $J$ 2017;26:324-35.

11. Fernandez M, Ferreira ML, Refshauge KM, et al. Surgery or physical activity in the management of sciatica: a systematic review and meta-analysis. Eur Spine J 2016;25:3495-512.

12. Machado GC, Maher CG, Ferreira PH, et al. Non-steroidal antiinflammatory drugs for spinal pain: a systematic review and metaanalysis. Ann Rheum Dis 2017;76:1269-78.

13. Enthoven WTM, Roelofs PD, Koes BW. NSAIDs for Chronic Low Back Pain. JAMA 2017;317:2327-8.

14. Saragiotto BT, Machado GC, Ferreira ML, et al. Paracetamol for low back pain. Cochrane Database Syst Rev 2016;6:CD012230.

15. Mathieson S, Maher CG, McLachlan AJ, et al. Trial of Pregabalin for Acute and Chronic Sciatica. N Engl J Med 2017;376:1111-20.

16. Abdel Shaheed C, Maher CG, Williams KA, et al. Efficacy and tolerability of muscle relaxants for low back pain: Systematic review and meta-analysis. Eur J Pain 2017;21:228-37.

17. Abdel Shaheed C, Maher CG, Williams KA, et al. Efficacy, Tolerability, and Dose-Dependent Effects of Opioid Analgesics for Low Back Pain: A Systematic Review and Meta-analysis. JAMA Intern Med 2016;176:958-68.

18. Saragiotto BT, Maher CG, Yamato TP, et al. Motor Control Exercise for Nonspecific Low Back Pain: A Cochrane Review. Spine 2016;41:1284-95.

19. Macedo LG, Saragiotto BT, Yamato TP, et al. Motor control exercise for acute non-specific low back pain. Cochrane Database Syst Rev 2016;2:CD012085.

20. Saragiotto BT, Maher CG, Yamato TP, et al. Motor control exercise for chronic non-specific low-back pain. Cochrane Database Syst Rev 2016;1:CD012004.

21. Cherkin DC, Anderson ML, Sherman KJ, et al. Two-Year Follow-up of a Randomized Clinical Trial of Mindfulness-Based Stress Reduction 
vs Cognitive Behavioral Therapy or Usual Care for Chronic Low Back Pain. JAMA 2017;317:642-4.

22. Gatchel RJ, Rollings KH. Evidence-informed management of chronic low back pain with cognitive behavioral therapy. Spine $J$ 2008;8:40-4

23. Qaseem A, Wilt TJ, McLean RM, et al. Noninvasive Treatments for Acute, Subacute, and Chronic Low Back Pain: A Clinical Practice Guideline From the American College of Physicians. Ann Intern Med 2017;166:514-30.

24. Van Wambeke P, Desomer A, Ailiet L, et al. Low back pain and radicular pain: assessment and management. KCE Report 2017:287.

25. Almeida M, Saragiotto B, Richards B, et al. Primary care management of non-specific low back pain: key messages from recent clinical guidelines. Med J Aust 2018;208:272-5.

26. Hurley J, O'Keeffe M, O'Sullivan P, et al. Effect of education on nonspecific neck and low back pain: A meta-analysis of randomized controlled trials. Man Ther 2016;23:e1-e2.

27. Moseley GL. Evidence for a direct relationship between cognitive and physical change during an education intervention in people with chronic low back pain. Eur J Pain 2004;8:39-45.

28. Moseley GL, Nicholas MK, Hodges PW. A randomized controlled trial of intensive neurophysiology education in chronic low back pain. Clin $J$ Pain 2004;20:324-30.

29. Moseley G, Butler D. Explain pain supercharged. Adelaide, Australia: Noigroup publications, 2017.

30. Sharma S, Pathak A, Jensen MP. Words that describe chronic musculoskeletal pain: implications for assessing pain quality across cultures. J Pain Res 2016;9:1057-66.

31. Eldridge SM, Chan CL, Campbell MJ, et al. CONSORT 2010 statement: extension to randomised pilot and feasibility trials. BMJ 2016;355:i5239.

32. Chan AW, Tetzlaff JM, Gøtzsche PC, et al. SPIRIT 2013 explanation and elaboration: guidance for protocols of clinical trials. BMJ 2013;346:e7586.

33. Chen WH, Revicki DA, Amtmann D, et al. Development and Analysis of PROMIS Pain Intensity Scale. Quality of Life Research 2012;20:18.

34. Bardin LD, King P, Maher CG. Diagnostic triage for low back pain: a practical approach for primary care. Med J Aust 2017;206:268-73.

35. Billingham SA, Whitehead AL, Julious SA. An audit of sample sizes for pilot and feasibility trials being undertaken in the United Kingdom registered in the United Kingdom Clinical Research Network database. BMC Med Res Methodol 2013;13:104.

36. Mengoni SE, Gates B, Parkes G, et al. Wordless intervention for people with epilepsy and learning disabilities (WIELD): a randomised controlled feasibility trial. BMJ Open 2016;6:e012993.

37 Central Bureau of Statistics, Nepal. National Planning Commission Secretariat. ed. National Population and Housing Census 2011 (National Report). Kathmandu: Government of Nepal, 2012.

38. Yamato T, Maher C, Saragiotto B, et al. The TIDieR Checklist Will Benefit the Physical Therapy Profession. J Orthop Sports Phys Ther 2016;46:402-4.

39. Hoffmann TC, Glasziou PP, Boutron I, et al. Better reporting of interventions: template for intervention description and replication (TIDieR) checklist and guide. BMJ 2014;348:g1687.

40. Butler DS, Moseley GL. Explain Pain 2nd Edn: Noigroup Publications, 2013.

41. Moseley GL, Butler DSB. Explain Pain Handbook: Protectometer, 2015.

42. Moseley GL, Butler DS. Fifteen Years of Explaining Pain: The Past, Present, and Future. J Pain 2015;16:807-13.

43. Clarke CL, Ryan CG, Martin DJ. Pain neurophysiology education for the management of individuals with chronic low back pain: systematic review and meta-analysis. Man Ther 2011;16:544-9.

44. Traeger AC, Hübscher M, Henschke N, et al. Effect of Primary Care-Based Education on Reassurance in Patients With Acute Low Back Pain: Systematic Review and Meta-analysis. JAMA Intern Med 2015;175:733-43.

45. Linton SJ, McCracken LM, Vlaeyen JW. Reassurance: help or hinder in the treatment of pain. Pain 2008;134(1-2):5-8.

46. Koes BW, van Tulder M, Lin CW, et al. An updated overview of clinical guidelines for the management of non-specific low back pain in primary care. Eur Spine J 2010;19:2075-94.
47. Traeger AC, O'Hagan ET, Cashin A, et al. Reassurance for patients with non-specific conditions - a user's guide. Braz J Phys Ther 2017;21:1-6.

48. Stableford S, Mettger W. Plain language: a strategic response to the health literacy challenge. J Public Health Policy 2007;28:71-93.

49. Bernstein IA, Malik Q, Carville $S$, et al. Low back pain and sciatica: summary of NICE guidance. BMJ 2017;356:i6748.

50. Toward Optimized Practice (TOP) Low Back Pain Working Group. Evidence-informed primary care management of low back pain: clinical practice guideline. 2015 http://www.topalbertadoctors.org/ cpgs/885801.

51. National Institute for Health and Care Excellence. Low back pain and sciatica in over 16s: assessment and management. $2016 \mathrm{https} / / /$ www.nice.org.uk/guidance/ng59.

52. O'Connell NE, Cook CE, Wand BM, et al. Clinical guidelines for low back pain: A critical review of consensus and inconsistencies across three major guidelines. Best Pract Res Clin Rheumatol 2016;30.

53. Chiarotto A, Deyo RA, Terwee CB, et al. Core outcome domains for clinical trials in non-specific low back pain. Eur Spine $J$ 2015;24:1127-42.

54. Sharma S, Pathak A, Maharian R, et al. Psychometric properties of nepali versions of PROMIS short from measures of pain intensity, pain interference, pain behaviour, depressions, and sleep disturbance. The Journal of Pain 2018;19:S59.

55. Sharma S, Thibault P, Abbott JH, et al. Clinimetric properties of the Nepali version of the Pain Catastrophizing Scale in individuals with chronic pain. J Pain Res 2018;11:265-76.

56. Sharma S, Pathak A, Abbott JH, et al. Measurement properties of the Nepali version of the Connor Davidson resilience scales in individuals with chronic pain. Health Qual Life Outcomes 2018;16:56.

57. Sharma S, Palanchoke J, Reed D, et al. Translation, cross-cultural adaptation and psychometric properties of the Nepali versions of numerical pain rating scale and global rating of change. Health Qual Life Outcomes 2017;15:236.

58. Sharma S, Palanchoke J, Abbott JH. Cross-Cultural Adaptation and Validation of the Nepali Translation of the Patient-Specific Functional Scale. J Orthop Sports Phys Ther 2018-1-23.

59. Geraghty AW, Stanford R, Little P, et al. Using an internet intervention to support self-management of low back pain in primary care: protocol for a randomised controlled feasibility trial (SupportBack). BMJ Open 2015:5:e009524.

60. Shanyinde M, Pickering RM, Weatherall M. Questions asked and answered in pilot and feasibility randomized controlled trials. BMC Med Res Methodol 2011;11:117.

61. Abbott $\mathrm{JH}$. The distinction between randomized clinical trials (RCTs) and preliminary feasibility and pilot studies: what they are and are not. J Orthop Sports Phys Ther 2014;44:555-8.

62. Pinheiro MB, Ho KK, Ferreira ML, et al. Efficacy of a Sleep Quality Intervention in People With Low Back Pain: Protocol for a Feasibility Randomized Co-Twin Controlled Trial. Twin Res Hum Genet 2016;19:492-501.

63. Borkovec TD, Nau SD. Credibility of analogue therapy rationales. $J$ Behav Ther Exp Psychiatry 1972;3-257-60.

64. Pathak A, Sharma S, Jensen MP. The Utility and Validity of Pain Intensity Rating Scales for use in Developing Countries. Pain Reports. In Press. 2018

65. Dworkin RH, Jensen MP, Gould E, et al. Treatment satisfaction in osteoarthritis and chronic low back pain: the role of pain, physical and emotional functioning, sleep, and adverse events. J Pain 2011;12:416-24.

66. Dworkin RH, Turk DC, Farrar JT, et al. Core outcome measures for chronic pain clinical trials: IMMPACT recommendations. Pain 2005;113(1-2):9-19.

67. Farrar JT, Young JP, LaMoreaux L, et al. Clinical importance of changes in chronic pain intensity measured on an 11-point numerical pain rating scale. Pain 2001;94:149-58.

68. Crane HM, Van Rompaey SE, Dillingham PW, et al. A single-item measure of health-related quality-of-life for HIV-infected patients in routine clinical care. AIDS Patient Care STDS 2006;20:161-74. 